\title{
A mismatch that works
}

\begin{abstract}
Mismatched MHC alleles between donor and recipient represent a major barrier for adoptive T-cell therapy for immunodeficient patients and, in the case of haematopoietic stem-cell transplantation (HSCT), can cause graft rejection and graft-versus-host disease (GVHD). Now, Zakrzewski et al. show that allogeneic, MHC-mismatched T-cell precursors can be transferred to immunodeficient recipients irrespective of MHC restriction to give rise to functional mature $\mathrm{T}$ cells that are tolerant to host and donor antigens and, in addition, have beneficial graft-versus-tumour activity.

Notch-based culture systems provide an elegant means to generate T-cell lineages from haematopoietic cells ex vivo. Using such a system, T-cell precursors were generated by culturing haematopoietic stem cells $\left(\mathrm{LIN}^{-} \mathrm{SCA}^{+}{ }^{+} \mathrm{KIT}^{\mathrm{hi}}\right)$ derived from C57BL/6 mice with OP9 bone-marrow stromal cells that
\end{abstract}

express the Notch ligand DLL1 (delta-like ligand 1), soluble FMS-like tyrosine kinase 3 (FLT3) ligand and interleukin-7. To assess their immunotherapeutic potential, these allogeneic T-cell precursors were transferred to lethally irradiated $\mathrm{BALB} / \mathrm{c}$ mice following syngeneic HSCT.

In mice that received allogeneic $\mathrm{T}$-cell precursors, $\mathrm{T}$ cells and natural killer cells reconstituted more efficiently in the early period posttransplant (days 14 to 28) compared with mice that underwent HSCT alone. In addition, small numbers of myeloid and dendritic cells developed from the transferred precursor cells. No clinical or subclinical features of GVHD were detected in these mice and similar enhancement of leukocyte reconstitution was observed in sublethally irradiated BALB/c mice that received allogeneic $\mathrm{T}$-cell precursors in the absence of HSCT.

Using a mixed leukocyte reaction, it was shown that the mature $\mathrm{T}$ cells that developed in recipient BALB/c mice from allogeneic precursors were not reactive to host or donor antigens, but were reactive to third-party antigens, indicating that allogeneic T-cell precursors underwent negative selection on MHC molecules from both backgrounds, due to the antigen-presenting-cell chimerism established in the host. By contrast, positive selection was restricted to host MHC molecules.

Further analysis showed that transferred allogeneic T-cell precursors could reduce the progression of tumours that were strongly immunogenic and thereby enhance host survival (similar to syngeneic T-cell precursors). In addition, transfer of genetically engineered T-cell precursors that expressed a chimeric antigen receptor with an extracellular portion that targets human CD19 and an intracellular portion that consists of the $\mathrm{CD} 3 \zeta$ chain, resulted in additional antitumour activity against human CD19-expressing tumours, without any observed adverse effects.

So, the data show that allogeneic T-cell precursors generated ex vivo can be transferred to immunodeficient MHC-mismatched recipients and give rise to mature, dual tolerant (host and donor) T cells with antitumour activity, and that the function of these cells can be further enhanced by genetic engineering. Therefore, this study provides us with the first evidence of the possibility of 'off-the-shelf' T-cell immunotherapy.

\section{Olive Leavy}

ORIGINAL RESEARCH PAPER Zakrzewski, J. L. et al. Tumor immunotherapy across MHC barriers using allogeneic T-cell precursors. Nature Biotech. 30 March 2008 (doi:10.1038/nbt1395) 